Revue internationale P.M.E.

Économie et gestion de la petite et moyenne entreprise

\title{
Le financement des PME dans leur espace de transaction : le relâchement de l'hypothèse de rationnement du crédit
}

\section{Sylvie Cieply et Bob Hancké}

Volume 11, numéro 4, 1998

URI : https://id.erudit.org/iderudit/1009053ar

DOI : https://doi.org/10.7202/1009053ar

Aller au sommaire du numéro

Éditeur(s)

Presses de l’Université du Québec

ISSN

0776-5436 (imprimé)

1918-9699 (numérique)

Découvrir la revue

Citer cette note

Cieply, S. \& Hancké, B. (1998). Le financement des PME dans leur espace de transaction : le relâchement de l'hypothèse de rationnement du crédit. Revue internationale P.M.E., 11(4), 95-104. https://doi.org/10.7202/1009053ar

\section{Résumé de l'article}

La documentation traitant du financement des petites et moyennes entreprises fait souvent état de l'existence d'un rationnement du crédit se produisant à l'encontre de cette catégorie d'entreprises. Cette hypothèse trouve un support formel dans la théorie du rationnement du crédit. Les résultats des travaux empiriques ayant testé cette hypothèse nous amènent cependant à nous interroger sur sa validité et à proposer une voie de recherche permettant de concilier les résultats des études théoriques et empiriques menées sur le thème " rationnement du crédit et PME ». Dans cette note de recherche, nous montrons en effet que l'appréhension de la PME dans son espace de transaction permet de relâcher l'hypothèse d'un rationnement du crédit pour une majorité de ces entreprises. L'existence de relations interentreprises constitue en effet un canal pour des flux informationnels susceptibles de résoudre en partie les asymétries d'information qui causent les phénomènes de rationnement. Ces relations réduisent également les risques supportés parles créanciers. Elles peuvent finalement se traduire par des flux financiers qui complètent les apports des établissements de crédit.
Ce document est protégé par la loi sur le droit d'auteur. L'utilisation des services d’Érudit (y compris la reproduction) est assujettie à sa politique d'utilisation que vous pouvez consulter en ligne.

https://apropos.erudit.org/fr/usagers/politique-dutilisation/ 


\title{
Notes de recherche
}

\section{Le financement des PME dans leur espace de transaction: le relâchement de l'hypothèse de rationnement du crédit}

\author{
Sylvie CIEPLY \\ Université de Caen \\ Bob HANCKÉ \\ Wissenschaftszentrum für sozialforschung de Berlin
}

\section{MOTS CLÉS}

\section{PME - Rationnement du crédit - Information Risque - Financement interentreprises}

\begin{abstract}
RÉSUMÉ
La documentation traitant du financement des petites et moyennes entreprises fait souvent état de l'existence d'un rationnement du crédit se produisant à l'encontre de cette catégorie d'entreprises. Cette hypothèse trouve un support formel dans la théorie du rationnement du crédit. Les résultats des travaux empiriques ayant testé
\end{abstract}

\section{LES AUTEURS}

Sylvie Cieply est maître de conférences à l'IUP Banque-Assurance de l'Université de Caen. Elle exerce plus précisément les fonctions de responsable du Département opérations bancaires et financières internationales. Elle a obtenu en 1997 un doctorat en sciences économiques à l'Université Lumière-Lyon II sur le thème des réponses de la théorie du financement face aux spécificités financières des PME. Elle est maintenant membre du GEMMA - LERE à l'Université de Caen. Adresse: IUP Banque-assurance, esplanade de la Paix, 14032 Caen Cedex. Courriel : cieply@iupba.unicaen.fr

Bob Hancké est chercheur au Wissenschaftszentrum für sozialforschung de Berlin. II travaille dans l'équipe dirigée par David Soskice qui étudie les changements économiques. Dans ce cadre, Bob Hancké réalise, entre autres, des travaux sur la réorganisation de l'industrie française dans les années 1980. Adresse: WZB, Reichpietschufer 50, 10785 Berlin. Courriel: hancke@medea.wz-berlin.de 
cette hypothèse nous amènent cependant à nous interroger sur sa validité et à proposer une voie de recherche permettant de concilier les résultats des études théoriques et empiriques menées sur le thème "rationnement du crédit et PME ». Dans cette note de recherche, nous montrons en effet que l'appréhension de la PME dans son espace de transaction permet de relâcher l'hypothèse d'un rationnement du crédit pour une majorité de ces entreprises. L'existence de relations interentreprises constitue en effet un canal pour des flux informationnels susceptibles de résoudre en partie les asymétries d'information qui causent les phénomènes de rationnement. Ces relations réduisent également les risques supportés par les créanciers. Elles peuvent finalement se traduire par des flux financiers qui complètent les apports des établissements de crédit.

\section{ABSTRACT}

Literature concerning small business financing often indicates the existence of a small business capital gap. This hypothesis can find a formal support in the theory of credit rationing. However, the empirical studies testing this hypothesis do not permit to accept it irrevocably. Thus, in order to conciliate these theoretical and empirical results, we propose to integrate in the analysis of small firms' access to credit the characteristics of their transaction space. We show that interfirms relations are a source of information for bankers. They make banking risk and informational asymmetries decrease. Finally, interfirms credit flows can complete banking credit flows.

\section{RESUMEN}

La literatura sobre la financiación de las pequeñas empresas, a menudo distingue la existencia de un capital gap produciéndose en el crédito de dicha categoria de empresas. Esta hipótesis encuentra un soporte formal en la teoria del racionamiento del crédito. Las caractéristicas intrinsecas de la pequeña y mediana empresa, por un lado, y las condiciones necesarias a la aparición de un racionamiento sobre el mercado por otro lado, conducen a una "sobre exposición" de esta categoria de firmas. Los resultados de trabajos empiricos que han demostrado, de manera o más menos directa esta hipótesis, nos llevan a interroganos, à la vez, sobre su validación (pertinencia) y sobre una otra possible via de investigacion que permite de conciliar los resultados de estudios teóricos y empiricos desarrollados sobre el tema del "racionamiento del crédito y pequeñas y medianas empresas". En este articulo demostramos que la aprehensión de la pequeña empresa dentro de su medio natural permite descartar la hipótesis de un racionamiento del crédito por una gran parte de dichas empresas.

\section{Introduction}

Le manque d'informations crédibles fournies par les petites entreprises, le risque important supporté par les créanciers sur ce segment de clientèle et la faible dotation de ces entreprises en actifs pouvant avoir valeur de garantie semblent, en théorie, surexposer les PME aux phénomènes de rationnement sur le marché du crédit (Binks, Ennew et Reed, 1992 ; Psillaki, 1995 ; Ross, 1996). Or, les travaux empiriques 
réalisés sur cette question ne confirment pas cette hypothèse. Les enquêtes d'opinions placent au second rang des préoccupations des dirigeants la disponibilité du crédit (Jasor, 1998 ; Bannock et Morgan, 1998 ; HMSO, 1991). Les travaux qui analysent le volume du crédit commercial pour approcher le rationnement du crédit ne relèvent aucune relation entre l'importance de cette variable et la taille de la firme (Petersen et Rajan, 1994). Enfin, l'identification des régimes de financement des entreprises françaises selon leur taille ne permet pas de conclure, lorsque l'on considère l'endettement total de la firme, à une situation plus difficile des PME sur le marché du crédit (Cieply et Paranque, 1998).

Comment donc concilier la démonstration de la surexposition des PME au rationnement du crédit et le constat empirique de la faible contrainte de ces entreprises? La réponse que nous proposons dans cette note de recherche est qu'une proportion importante de PME échappe au rationnement sur le marché du crédit en raison de leur inscription dans une organisation en réseau centrée sur une entreprise établie, a fortiori, une grande entreprise. En effet, le développement de relations entre entreprises fournit tout d'abord un canal pour des flux informationnels susceptibles de résoudre, dans une certaine mesure, les asymétries d'information qui causent, en grande partie, le rationnement du crédit. L'inscription des petites entreprises dans un espace de transaction centré sur une entreprise établie peut ensuite permettre aux banques, principalement commerciales, de réduire leur risque de contrepartie sur cette clientèle. Enfin, nous soulignons l'existence possible de financements interentreprises qui peuvent accompagner les stratégies de développement industriel ou local des entreprises établies et ainsi compléter le financement bancaire.

\section{Relations interentreprises, flux d'informations et financement bancaire des petites entreprises}

Le choix d'un partenaire non établi par une entreprise établie à la fois sur le marché du crédit et sur le marché des biens et services peut signaler à la banque la qualité de l'entreprise non établie. L'accord passé signale en effet la pertinence du domaine d'activité stratégique et des compétences de ces firmes.

Cette analyse concerne en premier lieu les fournisseurs des entreprises établies, en particulier les entreprises sous-traitantes. Une entreprise établie sélectionne en effet les fournisseurs qui lui offrent le meilleur rapport qualité-prix. Cet accord commercial peut, en conséquence, signaler aux investisseurs la compétitivité de l'entreprise non établie. Lorsque l'accord est passé entre une petite et une grande entreprise, ce signal est à la fois plus fort et plus formel dans la mesure où il nécessite au préalable, le plus souvent, la certification ou l'audit de la petite entreprise.

La certification constitue une procédure publique de reconnaissance de la qualité de la firme. Dans ce cadre normatif sont évaluées la façon dont un produit 
est réalisé et les ressources consacrées à l'organisation du contrôle de la qualité. La certification assure finalement aux grandes entreprises, mais également aux banques, l'aptitude d'une PME à ajuster sa production aux variations de la demande.

Tandis que beaucoup de grandes entreprises sélectionnent leurs sous-traitants sur la base unique de la certification ISO 9000, un nombre encore important de firmes se réfère aussi à un système d'audition de la qualité « maison », c'est-à-dire spécifique et privé. La grande entreprise envoie dans la PME une équipe composée de ses propres experts qui examinent tous les procédés intervenant dans la qualité, la livraison, la tarification ou les autres aspects jugés essentiels de l'activité de l'entreprise. Cette information donnée par des auditeurs expérimentés et réputés permet à la grande entreprise d'évaluer le potentiel à long terme du fournisseur. Malgré leur caractère privé, les audits peuvent également avoir une valeur informationnelle pour la banque. Le simple fait qu'un audit de qualité ait été réalisé et que, à la suite de cet audit, des relations interentreprises aient été engagées, constitue une bonne nouvelle sur la qualité de la firme. L'efficacité de ce mécanisme de signalement repose finalement sur la crédibilité et la notoriété de l'entreprise qui réalise l'audit. Cette condition apparaît en général respectée dans la mesure où ce sont les plus grandes entreprises industrielles qui ont conservé les audits de qualité individuels, cela même parmi les fournisseurs certifiés.

Cette analyse concerne également les clients des entreprises établies. Les entreprises établies recherchent des clients solvables susceptibles de les payer dans les meilleurs délais. Elles évaluent la solvabilité de leurs clients non seulement à partir des informations financières relatives à l'entreprise non établie, mais également à partir de l'étude de sa capacité à se développer, de manière pérenne, sur son marché. Le processus de sélection des grandes entreprises apparaît ainsi particulièrement efficace puisque la part des créances douteuses et litigieuses sur les créances totales est la moins importante dans ces firmes (Dietsch, 1998).

Enfin, l'existence d'un processus partenarial en matière de recherche et développement entre une grande entreprise et une petite firme non établie constitue une reconnaissance par la première de la compétence technologique de la seconde. Cet engagement atteste également de la valeur intrinsèque de revente de la petite entreprise non établie.

L'existence d'un accord de coopération entre une entreprise reconnue par le système bancaire, a fortiori, une grande entreprise, et une PME à la recherche de financements et de réputation sur le marché du crédit peut donc inciter la banque à financer cette dernière catégorie de firmes. L'entreprise établie sur le marché du crédit peut ainsi jouer le rôle d'un intermédiaire en information/réputation entre la PME non établie et le système bancaire. Ce transfert de réputation illustre particulièrement bien le modèle décrit par Ellis (1992) selon lequel la réputation ne doit 
pas obligatoirement être établie directement par les agents impliqués dans la relation considérée. La banque peut en effet obtenir des informations et se faire une opinion sur une entreprise en observant les interactions de cette dernière avec une autre entreprise dont la réputation sur le marché du crédit est établie. Ce mécanisme de transfert de réputation n'implique pas l'existence d'une relation formelle entre l'entreprise établie et l'établissement de crédit. Il suffit que la notoriété de l'entreprise établie soit reconnue par le banquier de l'entreprise non établie.

Ainsi, la coopération interentreprises permettrait, en signalant aux banques la qualité de la petite entreprise, de réduire l'occurrence d'un phénomène d'antisélection sur le marché du crédit. Les mécanismes évoqués jusqu'à présent ne permettent pas cependant de démontrer un quelconque effet de l'existence d'une relation interentreprises sur l'importance des risques d'aléas de moralité et d'opportunisme.

Quand l'entreprise non établie est le fournisseur de l'entreprise établie, le risque d'aléas de moralité peut être réduit du fait des contrôles de qualité réguliers que réalise la firme cliente. Quant au risque d'opportunisme, il apparaît, dans ce contexte, minimisé du fait de la stabilisation du chiffre d'affaires de la petite entreprise qui rend moins crédible une déclaration de résultat sous-évaluée et qui, en conséquence, dissuade son dirigeant de tricher.

Quand l'entreprise non établie est cliente de l'entreprise établie ou lorsqu'un accord de partenariat en recherche et développement a été passé entre ces deux firmes, le risque respectivement de non-renouvellement du contrat de vente ou du retrait de la grande entreprise du projet de R-D incite la firme non établie à ne pas prendre trop de risques de manière à rester solvable. L'entreprise non établie n'a pas, non plus, intérêt à sous-évaluer ses résultats dans la mesure où cela pourrait effrayer son partenaire industriel et l'amener à cesser sa collaboration sauf s'il existe une collusion entre l'entreprise établie et l'entreprise non établie. Dans ce cas particulier, l'entreprise établie risque de perdre sa notoriété, ce qui limite l'occurrence d'un tel comportement ${ }^{1}$. Le risque d'opportunisme de la part des entreprises non établies apparaît ainsi pouvoir être écarté.

Au total, l'intégration des petites entreprises dans des systèmes centrés sur une ou des entreprises établies, a fortiori, des grandes entreprises, permet à la banque de déléguer l'analyse et le suivi industriel de la petite entreprise à ces firmes. La sélection et le contrôle seraient ainsi délégués aux entreprises établies qui deviennent ainsi des intermédiaires en information. L'appartenance des petites entreprises à une ou des organisations en réseau permet également, sous certaines conditions, de diminuer l'exposition de la banque au risque de contrepartie sur cette clientèle.

1. Sur ce point, on peut penser que la notoriété de l'entreprise établie dépend, en grande partie, de sa capacité à ne pas entrer dans ce type de relation avec une entreprise non établie. 


\section{Relations interentreprises, risque et financement bancaire des petites entreprises}

L'existence de relations commerciales suivies entre une PME non établie et une entreprise établie, a fortiori, une grande entreprise, permet la diminution du risque supporté par le banquier sur cette première catégorie d'entreprises en raison de la relative stabilisation de leur activité. L'existence de ces relations interentreprises donne en effet aux entreprises non établies une relative assurance de débouchés qui permet de limiter les incertitudes du banquier quant à la vraisemblance du chiffre d'affaires anticipé et annoncé par le dirigeant de l'entreprise non établie.

Ce constat s'impose même dans le cadre d'un accord de sous-traitance compte tenu des transformations enregistrées par ce type de relations depuis la fin des années 1970 (Baudry, 1995). Au lieu de reposer sur un nombre important de fournisseurs produisant des pièces particulières, les grandes entreprises sont entrées en partenariat avec un nombre limité de sous-traitants appelés à produire des fonctions entières du produit final. Les petites entreprises ne doivent pas alors seulement offrir des produits à bas prix : elles doivent adopter une démarche qualité. Dans ce contexte, le donneur d'ordres a tendance à s'engager, sur une période déterminée, à ne pas modifier sa politique de prix, à ne pas changer de fournisseurs et à ne pas fabriquer lui-même la pièce réalisée par le sous-traitant. La grande entreprise garantit ainsi l'amortissement des investissements réalisés et assure à la petite un flux de chiffre d'affaires relativement régulier ${ }^{2}$. Enfin, pour éviter toute mise en responsabilité lorsque, en cas de diminution brutale du niveau d'activité, le donneur d'ordres réduit le volume de ses commandes, les grandes entreprises exigent de leurs fournisseurs qu'ils diversifient leur clientèle de manière à réduire leur risque de dépendance économique.

Au total, une fois prise en compte l'inscription des PME dans leur réseau productif, leurs marchés apparaissent moins incertains. Le risque PME apparaît dès lors considérablement réduit pour les banques commerciales qui peuvent finalement adosser leurs crédits aux créances portant sur l'entreprise établie ${ }^{3}$. Aux États-Unis, les crédits basés sur les actifs et, en France, l'escompte et les crédits Dailly sont des techniques de financement qui reposent sur l'existence de créances jugées de bonne qualité par l'établissement de crédit. L'existence de relations entre une PME non établie et une entreprise établie peut donc améliorer le pouvoir de négociation de la première en la rendant moins fragile à court terme aux yeux de son banquier, voire en lui ouvrant la possibilité d'être financée par la banque du partenaire établie.

2. Pour l'industrie automobile, le lecteur peut consulter Darréon et Façail (1993).

3. La fourniture de crédit ne dépasse pas $80 \%$ de la valeur des créances détenues par la firme et jugées recevables par la banque, c'est-à-dire émises par des entreprises de qualité supérieure. 
Dans certains cas où les relations entre PME et grandes entreprises sont particulièrement intenses, la grande entreprise peut intervenir pour faire profiter son partenaire de son pouvoir de négociation avec sa propre banque. La grande entreprise émet alors des billets à ordre que la PME fait escompter auprès de la banque de la grande entreprise et selon les conditions dont bénéficierait cette dernière (ChanelReynaud et Cieply, 1998). À long terme, cependant, la stabilité du système peut être remise en cause par l'évolution de la situation ou de la politique commerciale de l'entreprise établie. Ainsi, l'existence de relations interentreprises ne permettrait une réduction des risques que sur le marché du crédit à court terme, ce qui apparaît cohérent avec le constat d'un accès plus difficile des PME aux crédits à moyen et long terme (Cieply et Paranque, 1998). Enfin, l'existence d'accords de coopération peut, sous certaines conditions, se traduire par un apport de financement interentreprises susceptible de venir compléter le financement bancaire des PME.

\section{Les financements interentreprises: un complément au financement bancaire des PME}

Les relations partenariales qui s'établissent entre entreprises mettent en œuvre, sous certaines conditions, des ressources financières.

L'apport de financement à une petite entreprise peut, tout d'abord, s'inscrire dans un partenariat de type industriel. L'existence de relations de crédit rend en effet plus efficace un accord de coopération industriel (Déi Ottati, 1994). Le financement interentreprises réduit les risques de défaut du partenaire quand la demande augmente. Les entreprises sécurisent ainsi leur approvisionnement ou leur distribution. En cas d'effondrement de la demande, elles sont par ailleurs incitées à choisir une stratégie coopérative. La faillite du partenaire est en effet sous-optimale dans la mesure où elle induit non seulement des frais de recherche d'un nouveau partenaire, mais également la perte du financement octroyé. L'existence d'un financement interentreprises permet donc de stabiliser la relation industrielle. Il consolide le partenariat en créant des obligations réciproques supportées par « des engagements crédibles constitutifs d'une assurance mutuelle contre les incertitudes du marché » (Déi Ottati, 1994, p. 537).

Le financement interfirmes le plus répandu, particulièrement en France, est le crédit fournisseur qui constitue le premier moyen de financement mobilisé par les entreprises en création et le second, après le crédit bancaire, pour les très petites et les petites entreprises (Julien, 1994).

Le financement interfirmes peut également consister en des prises de participation dans le capital du partenaire. Le montant des participations croisées entre entreprises (participations au capital, comptes courants d'associés, obligations convertibles, prêts participatifs à bons de souscription d'action) a ainsi fortement 
augmenté en 20 ans. Ce mouvement résulte principalement des prises de participation des entreprises dans le capital d'autres entreprises non cotées, des PME pour la plupart. La proportion d'actions cotées détenues par les entreprises a en effet stagné sur cette période ( $20 \%$ en 1977 contre $21 \%$ fin 1992) alors que la proportion d'actions non cotées détenues par ces mêmes entreprises est passée de $42 \%$ en 1977 à $65 \%$ en 1992 (Babeau, 1994).

D'autres interventions des grandes entreprises peuvent être interprétées comme un moyen indirect de financer les PME partenaires. Il s' agit notamment de la fourniture gratuite d'équipements, de technologies ou de services (Beuscart, 1996; Rey, 1996). Ces investissements, dont la valeur est relativement faible à l'échelle de la grande entreprise, sont en revanche très conséquents à l'échelle de la petite. Certaines grandes entreprises mettent ainsi à la disposition des PME partenaires leur réseau commercial à l'étranger. Elles offrent également à ces firmes un accès privilégié à des services fiscaux ou techniques propres aux grandes entreprises et excessivement coûteux, voire non accessibles par de petites structures.

L'engagement des grandes entreprises peut également être d'ordre social et s'inscrire dans un partenariat de type local. La grande entreprise finance alors des PME implantées sur ses principaux sites industriels, particulièrement là où son activité décline. La grande entreprise accompagne ainsi son désengagement, parfois brutal, sur une zone géographiquement déterminée ${ }^{4}$. Après une première action, souvent qualifiée d'opération commando, il n'est pas rare que les interventions prennent une forme plus durable. Dans les deux cas, l'instrument financier utilisé est le crédit à moyen ou à long terme distribué par des sociétés de reconversion, filiales des grands groupes. Ces crédits, d'un montant en France de 10000 à 120000 francs par emploi créé dans la limite de 300000 francs, sont contractés à des taux inférieurs au taux du marché, sans apport de garantie et à remboursement différé. Certaines sociétés financières transforment ces crédits en subventions si l'entreprise embauche des salariés de l'entreprise créancière. Enfin, ces sociétés privilégient également les opérations d'essaimage qui permettent à la fois de réduire leur masse salariale et de mener une mission de développement local. Dans ce cadre, les grandes entreprises accompagnent administrativement, voire stratégiquement, l'entreprise financée.

Ainsi, sous les conditions précédemment définies, l'entreprise établie peut compléter l'offre bancaire, voire se substituer plus ou moins momentanément à cette offre. Il se produit alors un phénomène de double intermédiation puisque le crédit irrigue les petites entreprises non établies en partie par l'intermédiaire des entreprises établies avec qui elles sont en relation (Déi Ottati, 1994). Une fois l'entreprise établie

4. Ce type de financement est ainsi qualifié de «conscience money» par la Commission européenne (DG XXIII, 1988). 
sur son marché, on peut supposer que le financement bancaire prend naturellement le relais des financements interentreprises. La coexistence pendant une période plus ou moins longue d'un financement interentreprises et d'un financement bancaire permet à la banque d'observer l'entreprise cible et de construire avec cette dernière un espace commun de communication basée sur la réputation ${ }^{5}$.

\section{Conclusion}

L'existence de relations industrielles ou commerciales entre une petite entreprise non établie et une entreprise établie, a fortiori, une grande entreprise, apparaît donc pouvoir réduire l'exposition des premières au rationnement du crédit. L'existence de ces relations signale en effet aux banques la qualité de l'entreprise non établie. Elle réduit le risque encouru par la banque dans l'octroi de crédit à court terme. Enfin, ces relations peuvent, dans certains cas, s'accompagner de financements interentreprises qui complètent ou se substituent momentanément aux financements bancaires. Au terme de cette étude, il apparaît donc que le rationnement du crédit resterait un phénomène circonscrit aux petites entreprises qui n'entretiennent pas de relations étroites avec d'autres entreprises soit, selon les résultats de l'enquête PMI 90, moins de $20 \%$ des PME-PMI de France (Algoe, 1989). Pour ces entreprises, une solution institutionnelle s'organise en France autour de l'activité de la Banque de développement des PME laquelle fournit des financements à moyen et à long terme et garantit les crédits bancaires à court et à long terme que contractent ces entreprises particulièrement lors des épisodes les plus délicats de leur existence à savoir la création, l'innovation, la croissance et la transmission.

\section{Bibliographie}

Algoe (1989), PMI 90 : vers la compétitivité globale, Paris, Economica.

BABEAU, A. (1994), « Qui possède les entreprises en France ? », Le Monde, mardi 11 octobre, p. IV.

BAnNock, G. et Morgan, V.E. (1998), Banks and Small Business : An International Perspective, The Forum of Private Business, The National Federation of Independent Business, Londres.

BAUDRY, B. (1995), L'économie des relations interentreprises, Paris, La Découverte, 125 p.

BEUSCART, F. (1996), « Passerelle : les missionnaires du temps partagé », Liaisons Sociales le mensuel, janvier, p. 28-29.

5. La banque est alors le financeur secondaire de la petite entreprise. Elle gère cependant son compte courant, ce qui permet de constituer une information privée sur la petite entreprise (Nakaruma, 1993). 
BINKS, M., C. ENNEW et G. REED (1992), «Information asymmetries and the provision of finance to small firms », International Small Business Journal, vol. 11, no 1, p. 35-46.

Chanel-Reynaud G. et S. CiePly (1998), « Le financement des P.M.E. en France : d'un modèle à l'autre », Table Ronde "Finance et Industrie », Lyon, 26 mars.

CIEPLY, S. et B. PARANQUE (1998), «Le rationnement des petites entreprises sur le marché du crédit : mythe ou réalité ?», Banque et Marchés, $\mathrm{n}^{\circ}$ 33, mars-avril, p. 5-17.

DARRÉON, J-L. et S. FAIÇAL (1993), «Les enjeux des partenariats stratégiques entre grandes entreprises et P.M.E. », Revue française de gestion, septembre-octobre, p. 110-117.

DÉI OTTATI, G. (1994), « Trust, interlinking transactions and credit in the industrial district », Cambridge Journal of Economics, vol. 18, p. 529-546.

DG XXIII (1988), Partnership Between Small and Large Firms, Proceedings of the Conference Held in Brussels, 13 et 14 juin, Londres, Graham et Trotman.

DiETSCH, M. (1998), « Atouts et handicaps du crédit commercial face au crédit bancaire », Revue d'Économie Financière, no 46, mars, p. 175-193.

Ellis, C. J. (1992), « Reputation», dans P. Newman, M. Milgate et J. Eatwell, The New Palgrave, Dictionnary of Money \& Finance, tome 3, p. 331-332.

HMSO (1991), Constraints on the Growth of Small Firms, Londres, HMSO.

JASOR, M. (1998), «Amélioration des relations entre banques et PME», Les Échos, 16-17 janvier, p. 19.

JULIEN, P.A. (1994), Les PME : Bilan et perspectives, Cap-Rouge, Presses Inter Universitaires ; Paris, Economica, 437 p.

MARCHESNAY, M. et P. A. JULIEN (1989), «The small business : as a transaction space », Cahier de recherche du GREPME, $\mathrm{n}^{\circ}$ 89/15, paru dans Entrepreneur \& Regional Development, vol. 2, p. 267-277.

NAKARUMA, L. I. (1993), « Recent research in commercial banking : information and lending », Financial Markets, Institutions \& Instruments, vol. 2, $\mathrm{n}^{\circ}$ 5, décembre p. 73-88.

PETERSEN, M. et R. RAJAN (1994), « The benefits of lending relationships : evidence from small business data », The Journal of Finance, vol. XLIX, $\mathrm{n}^{\circ}$ 1, mars, p. 3-37.

PSILLAKI, M. (1995), « Rationnement du crédit et PME : Une tentative de mise en relation », Revue internationale PME, vol. 8, $\mathrm{n}^{\text {os }} 3$-4, p. 67-87.

REY, F. (1996), «Citroën - Rennes dynamisme des petites entreprises », Liaisons Sociales le mensuel, mai, p. 24-25.

Ross, D. M. (1996), « The unsatisfied fringe in Britain, 1930s-80s », Business History, vol. $38, \mathrm{n}^{\circ} 3$, p. 11-26. 